\title{
Peritoneal dialysis \\ Local solutions save young lives
}

\author{
Simon J Davies ${ }^{1 *}$ \\ ${ }^{1}$ Faculty of Medicine and Health Sciences, Keele University, UK \\ *E-mail: s.j.davies@keele.ac.uk
}

Peritoneal dialysis has many advantages over haemodialysis in the treatment of acute kidney injury (AKI) in low resource settings. One limitation, however, is the availability of commercial dialysis fluid. Following the International Society of Peritoneal Dialysis AKI guidelines, a frontline hospital in Cameroon now shows, , that locally prepared fluids are safe and effective.

Refers to Palmer, D. et al. Peritoneal dialysis for AKI in Cameroon: commercial vs locallymade solutions. Perit. Dial. Int. 38, 246-250 (2018).

When I was at Guy's Hospital London, one of my teachers, Chisholm Ogg, often remarked that what he liked about peritoneal dialysis was that it could be done in a field. The relatively simple gravity-driven set-up that is not dependent on an electricity supply combined with the usual lack of need for haemodynamic support, does indeed make peritoneal dialysis an attractive option for the treatment of acute kidney injury (AKI) in settings where resources are scarce. AKI is common in low income countries, especially in the young, often due to reversible causes such as malaria, snake bites, haemolytic-uraemic syndrome and infections. ${ }^{1,2}$ Given the ambition of the International Society of Nephrology (ISN) to prevent needless deaths from AKI by the year $2025,{ }^{3}$ a clear role exists for peritoneal dialysis without the need for major investment in infrastructure.

There is, however, one important infrastructural requirement - the availability of dialysis fluid that satisfies the regulatory standards of sterility, electrolyte and glucose composition. Making this fluid requires very substantial investment in specialised systems and is not commercially viable in many parts of the world. Although fluids can be shipped and 
transported by road this approach is prohibitively expensive for low-income countries, and can be exacerbated by additional official and unofficial tariffs at country borders.

Saving Young Lives is a collaborative venture between the International Society of Nephrology (ISN), International Society of Peritoneal Dialysis (ISPD), International Paediatric Nephrology Association (IPNA) and more recently EuroPD, that was set up with the objective of supporting clinical teams in the use of peritoneal dialysis to treat AKI in lowincome countries. ${ }^{4}$ From the start, this project recognised the need to source dialysis fluid, and partnered with the Sustainable Kidney Care Foundation, a public charity in the US devoted to providing treatment for kidney failure where non exists. The Foundation was able to pump-prime trained centres with a supply of dialysis fluid. Unfortunately, this plan proved not to be sustainable due to the high costs involved. An alternative approach was for centres to make their own dialysis solution by adding the more easily obtained $50 \%$ dextrose to commercially available intra-venous fluids such as Ringers lactate. This approach was described as an option in the ISPD 2014 guidelines for managing AKI, ${ }^{5}$ the first time that a kidney disease guideline has recommended an 'off label' treatment suitable for low resource settings in the absence of an alternative. Although it was clear in principle that such locally made solutions would work and indeed good anecdotal evidence suggests that they do, a systematic account of their use and safety in low-resource settings was lacking until now.

Palmer and colleagues, including supporters of the Mbingo Baptist Hospital in Cameroon now report the outcomes and complications of AKI treated with peritoneal dialysis, both using a commercial solution and following a period in which the researchers were unable to obtain commercial dialysis fluid, necessitating their switch to a locally-made solution. ${ }^{6}$ Their approach to local manufacture was to add $50 \%$ dextrose solution to Ringers lactate using standard aseptic techniques, and they used double-cuff Tenckhoff catheters in all patients. The electrolyte composition of locally made versus commercial solutions is summarised in the Table. The researchers treated a total of 68 patients between 2013 and 2017, including children and adults of whom 44 were under the age of 30 . Overall survival was $70 \%$ and survival rates did not differ between the 25 patients treated with commercial fluids (72\%) and the 43 treated with locally made solution (67\%). Of note, these rates are not dissimilar 
to those previously reported with use of peritoneal dialysis in this region of the world. ${ }^{7,8}$ The proportion of patients who developed peritonitis was identical in both groups at $16 \%$, which is impressively low given that patients received on average 52 dialysate exchanges each. When calculated as a rate, this equates to one peritonitis episode per 87 patient days for those receiving the commercial fluid compared to one peritonitis episode per 55 patient days for those receiving locally made dialysate. The proportion of patients who did not recover kidney function within 2 weeks was a little lower with the locally made solution (19\%) than with the commercial fluid (40\%).

Although the number of patients in this report is modest, the similarity of outcomes and complications in the two groups is reassuring. Of interest, the researchers - who should be proud of their overall outcomes - attribute much of their success to the training, establishment and acceptance of strict treatment protocols. To share their experiences they have created a YouTube video (https://youtu.be/-pB7kPT4gfc) as a teaching resource. As an exemplar of what Saving Young Lives is trying to achieve in the region, the success of the Mbingo team cannot be overstated. It is also an endorsement of the ISPD's decision to incorporate advice as to how local solutions can be used in their AKI guidelines. ${ }^{5}$

Lack of commercially available fluid is not, of course the only barrier to the treatment of AKI in resource challenged settings. Availability of peritoneal dialysis catheters, ideally soft rather than hard, that are affordable is a constant problem. Saving Young Lives has now trained nearly 200 doctors and nurses in sub-Saharan Africa, providing on-going support following training. The ability to use locally produced solutions undoubtedly adds value to this programme. However, the availability of dialysis solutions at an affordable price is the major factor holding back the use of peritoneal dialysis more widely in low-income countries, especially for the treatment of chronic kidney disease. ${ }^{9}$ What is now needed is a disruptive technology that enables the more sustainable production of dialysis fluid that is cheap and safe on a large scale

\section{References}


1. Cerdá, J., Bagga, A., Kher, V. \& Chakravarthi, R. M. The contrasting characteristics of acute kidney injury in developed and developing countries. Nat. Clin. Pract. Nephrol. 4, 138-153 (2008). [Au: just to let you know that we've published an updated version of this manuscript a few months ago: https://rdcu.be/bdBw2]

2. Susantitaphong, P. et al. World incidence of AKI: A meta-analysis. Clin. J. Am. Soc. Nephrol. 8, 1482-1493 (2013).

3. Mehta, R. L. et al. International Society of Nephrology's 0 by25 initiative for acute kidney injury (zero preventable deaths by 2025): a human rights case for nephrology. Lancet (London, England) 385, 2616-43 (2015).

4. Smoyer, W. E. et al. "Saving Young Lives" with acute kidney injury: the challenge of acute dialysis in low-resource settings. Kidney Int. 89, 254-256 (2016).

5. Cullis, B. et al. ISPD guidelines: Peritoneal dialysis for acute kidney injury. Perit. Dial. Int. 34, 494-517 (2014).

6. Palmer, D. et al. Peritoneal dialysis for AKI in Cameroon: commercial vs locally-made solutions. Perit. Dial. Int. 38, 246-250 (2018).

7. Kilonzo, K. G. et al. Outcome of acute peritoneal dialysis in northern Tanzania. Perit. Dial. Int. 32, 261-6 (2012).

8. Abdelraheem, M. et al. Outcome of Acute Kidney Injury in Sudanese Children -- An Experience from a Sub-Saharan African Unit. Perit. Dial. Int. 34, 526-533 (2014).

9. Caskey, F. J. et al. Global variation in renal replacement therapy for end-stage renal disease. Nephrol. Dial. Transplant 26, 2604-10 (2011).

\section{Competing interests statement}

S.D. is currently president of EuroPD and previous president of the International Society of Peritoneal Dialysis, organisations supporting Saving Young Lives. 
Table. Comparison of the electrolyte composition of commercial peritoneal dialysis fluid with those of intravenous fluids that can be used for the local manufacture of dialysate following the addition of $50 \%$ dextrose (courtesy Peritoneal Dialysis International ${ }^{5}$,)

Typical Composition of Commercially Available PD Fluid

\begin{tabular}{|c|c|c|c|c|c|c|c|c|c|}
\hline Type & $\mathrm{Na}^{+}$ & $\mathrm{K}^{+}$ & $\mathrm{Ca}^{2+}$ & $\mathrm{Mg}$ & Cl- & $\mathrm{HCO}_{3}^{-}$ & lactate & $\mathrm{pH}$ & osm. \\
\hline Stay-safe $1.5 \%$ & 132 & & 2.5 & 0.5 & 95 & & 40 & 5.5 & 344 \\
\hline Dianeal $1.5 \%$ & 132 & & 2.5 & 0.25 & 95 & & 35 & 5.2 & 344 \\
\hline \multicolumn{10}{|c|}{ Commercially Available Intravenous Fluids } \\
\hline Type of fluid & $\mathrm{Na}^{+}$ & $\mathrm{K}^{+}$ & $\mathrm{Ca}^{2+}$ & $\mathrm{Mg}$ & Cl- & $\mathrm{HCO}_{3}-$ & lactate & $\mathrm{pH}$ & osm. \\
\hline Hartmann's solution & 131 & 5 & 2.0 & & 111 & & 29 & 7.0 & 278 \\
\hline Ringer's lactate & 131 & 5 & 1.8 & & 112 & & 28 & 6.5 & 279 \\
\hline Plasmalyte B & 130 & 4 & 0 & 1.5 & 110 & 27 & & 7.4 & 273 \\
\hline
\end{tabular}

$\mathrm{Na}=$ sodium; $\mathrm{K}=$ potassium; $\mathrm{Ca}=$ calcium; $\mathrm{Mg}=$ magnesium; $\mathrm{Cl}=$ chlorine; $\mathrm{HCO}_{3}=$ bicarbonate; osm = osmolarity. 